\title{
Utilizing IEEE 802.16 for Aeronautical Communications
}

\author{
Max Ehammer, Thomas Gräupl and Elias Pschernig \\ University of Salzburg \\ Austria
}

\section{Introduction}

The future Air Traffic Management (ATM) concept shall be based on network centric operations, consequently on information sharing. In order to support such a vision not only a versatile and capable ground based communication network is necessary but also a network which includes the air to ground sub-networks which shall have sufficient capacity and capability. One such air to ground sub-network shall be established for the airport surface intended to be used by departing and arriving aircraft as well as by surface vehicles. This communication system is currently (2011) emerging and shall be called Aeronautical Mobile Airport Communications System (AeroMACS). AeroMACS shall be based on the IEEE 802.162009 standard (IEEE, 2009) and especially on the WiMAX Forum ${ }^{\mathrm{TM}}$ Mobile System Profile Specification rel1.0 v0.9 (WiMAX Forum, 2010). A draft profile has been developed and is being evaluated currently, e.g. in the EU research project SANDRA (SANDRA).

The IEEE 802.16-2009 standard (IEEE, 2009) specifies the air interface of combined fixed and mobile point to multipoint broadband wireless access systems with the possibility to support different services. The standard specifies the Medium Access Control (MAC) and the Physical (PHY) layer, where the MAC is capable to support multiple PHY specifications applicable to a specific operational environment. Figure 1 depicts the protocol reference model of the IEEE 802.16 standard. The Service Specific Convergence Sub-layer (CS) accepts higher layer data protocol units (PDUs) via the CS service access point (SAP). Thereby, the CS classifies each higher layer PDU according to available policies and maps each higher layer PDU to a so called service flow identifier. The IEEE 802.16 standard provides multiple CS specifications in order to provide interfaces for a variety of higher layer protocols. The MAC Common Part Sub-layer (CPS) provides the core functionality for data exchange via the wireless medium. A separate security sub-layer is also available. Generally, the IEEE 802.16-2009 standard provides a large amount of options. Thereby, different options may fit better for certain use cases than others. Due to the large amount of options it is merely impossible to be interoperable among different vendors based on the sole standard. Additional documentation and specification is necessary. This task has been conducted by the WiMAX Forum ${ }^{\mathrm{TM}}$. This group specifies so called "WiMAX profiles" where a selected set of options from the IEEE 802.16 standard is qualified for such a profile.

The WiMAX Forum ${ }^{\mathrm{TM}}$ has been established in June 2001 and is an industry led nonprofit organization. The purpose of the WiMAX forum ${ }^{\mathrm{TM}}$ is to certify compatibility and interoperability of broadband wireless products based on the IEEE 802.16 standard. In such a 
way rapid introduction of technology and market competition shall be enforced. The WiMAX Forum $^{\mathrm{TM}}$ has many members comprising the majority of operators and equipment vendors.

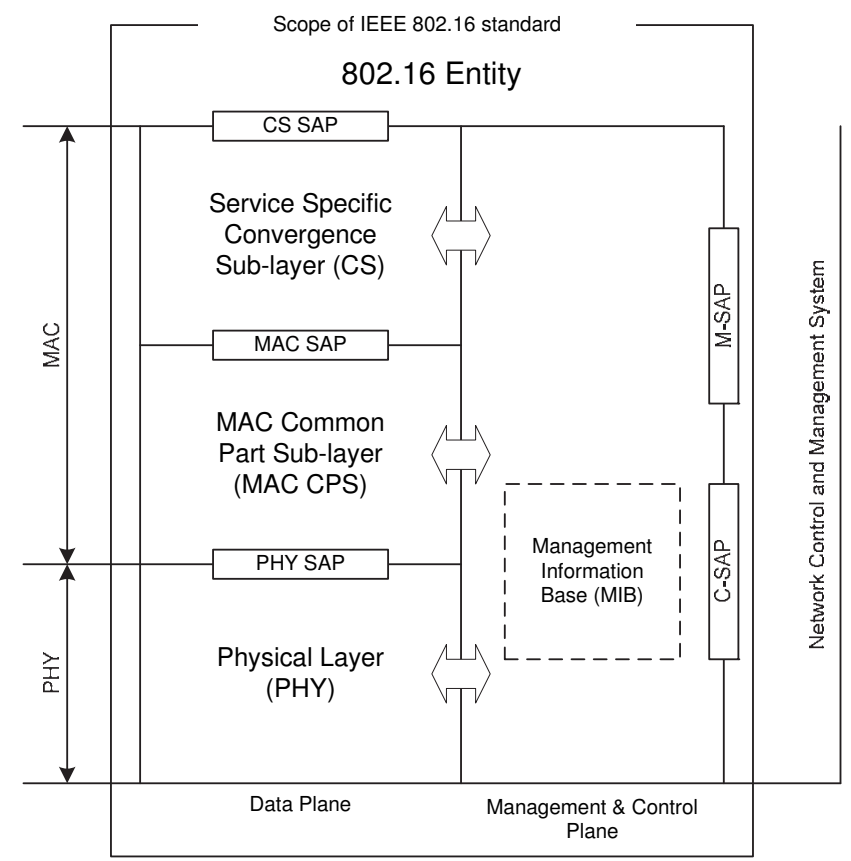

Fig. 1. The IEEE 802.16 protocol reference model.

The WiMAX Forum is organized into several working groups, one of them is the Technical Working Group (TWG), which develops technical product specifications and certification test suites for the air interface based on the OFDMA PHY. Such specifications are complementary to the IEEE 802.16 standards in order to achieve interoperability and certification of Mobile Stations and Base Stations conforming to the 802.16 standards. The TWG has produced a "Mobile System Profile Specification" which determines mandatory and optional functions.

Sub-chapter 2 gives an overview of selected AeroMACS profile items with some explanations. Sub-chapter 3 discusses the possibilities to run IPv6 over AeroMACS. Subchapter 4 summarizes the outcome of the data traffic load analysis conducted during the course of the SANDRA project. Sub-chapter 5 shows selected results of the medium access performance analysis. At the end concluding remarks finalize this chapter.

\section{AeroMACS profile overview}

The WiMAX Forum ${ }^{\mathrm{TM}}$ Mobile System Profile Specification (WiMAX, 2010) represents a subset of the IEEE 802.16 standard (IEEE, 2009). Currently (2011) a draft profile for AeroMACS is being specified through standardisation bodies such as EUROCAE and RTCA. This draft profile is further evaluated by members of the SESAR Joint Undertaking and by members of the SANDRA project (SANDRA, 2011). The tentative AeroMACS draft profile is further a subset of the WiMAX Forum ${ }^{\mathrm{TM}}$ Mobile System Profile Specification. 
Within this sub-chapter an overview of the core functionalities related to data exchange are given.

\subsection{Overview physical layer}

The Physical Layer (PHY) of the AeroMACS system shall be based on the OFDMA Physical Layer specification of the IEEE 802.16 standard with a channel bandwidth of $5 \mathrm{MHz}$. Thereby, the frame length shall be $5 \mathrm{~ms}$. As the PHY will be based on the "Common part TDD profile" (WiMAX 2009), the Downlink and Uplink portions can vary dependent on the system settings (cf. Figure 2).

The IEEE 802.16-2009 supports both Time Division Duplexing (TDD) and Frequency Division Duplexing (FDD) modes. However, AeroMACS shall be based on the TDD mode of operation. Reasons therefore are the dynamic allocation of Downlink (i.e. from Base Station to Mobile Station) and Uplink (i.e. from Mobile Station to Base Station) resources in order to efficiently support asymmetric Downlink (DL)/Uplink (UL) traffic, only a single channel is required which alleviates spectrum issues, and the TDD option is less complex.

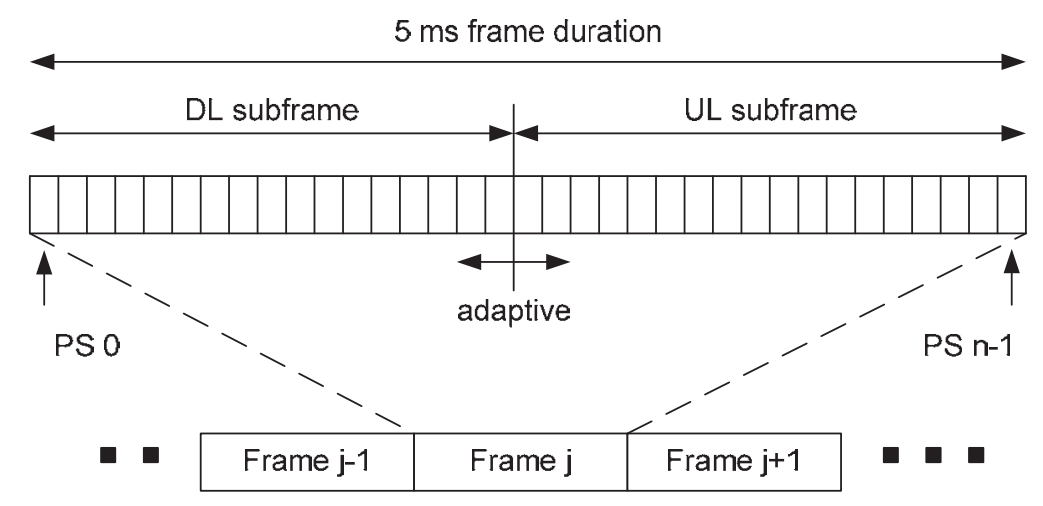

Fig. 2. AeroMACS frame with an adaptive DL/UL subframe width.

The DL subframe and the UL subframe consist of a number of OFDM symbols where a reasonable setting could be 29 OFDM symbols for the DL and 18 OFDM symbols for the UL. However, the individual setting is dependent on the service provider. Valid values can be taken from the WiMAX Forum ${ }^{\mathrm{TM}}$ Mobile System Profile Specification TDD Specific Part (WiMAX, 2009).

The standard supports multiple schemes for dividing the time and frequency resources among users, this may also be called sub-channelization. AeroMACS shall be based on the pseudo-random permutation for frequency diversity (i.e. PUSC). The available spectrum has to be utilized by the resource scheduler through an integer number of DL and UL slots, respectively. A slot is a logical $n \times m$ rectangle where $n$ is the number of sub-carriers and $m$ is the number of contiguous OFDM symbols. All slots, no matter which sub-channelization scheme is being used, contain 48 data symbols. Thereby, a DL slot consists of 2 OFDM symbols and 28 subcarriers. As the total usable amount of subcarriers is 420 for the DL, this results in 210 usable DL slots per $5 \mathrm{~ms}$ frame in the downlink direction (considering 28 OFDM symbols plus 1 OFDM symbol used for the DL Prefix). In contrast a UL slot consists of 3 OFDM symbols and 24 subcarriers. For the uplink direction the total usable amount of 
subcarriers is 408 , consequently there are 102 usable UL slots per $5 \mathrm{~ms}$ frame in the uplink direction (assuming 18 OFDM symbols).

Dependent on the coding and modulation scheme different throughput can be achieved. The modulation schemes are QPSK and 16 QAM for both directions as well as 64 QAM for the DL direction. 64 QAM is also being discussed as an option for the UL direction. Dependent on the robustness of the coding scheme different theoretical throughput values can be achieved.

\begin{tabular}{|c|c|c|c|c|c|c|}
\hline & \multicolumn{3}{|c|}{ DL } & \multicolumn{3}{c|}{ UL } \\
\cline { 2 - 7 } & QPSK & 16 QAM & 64 QAM & QPSK & 16 QAM & (64 QAM) \\
\hline CC $1 / 2$ & 48 bits & 96 bits & 144 bits & 48 bits & 96 bits & (144 bits) \\
\hline CC $2 / 3$ & 64 bits & 128 bits & 192 bits & 64 bits & 128 bits & (192 bits) \\
\hline CC $3 / 4$ & 72 bits & 144 bits & 216 bits & - & - & - \\
\hline CC $5 / 6$ & 80 bits & 160 bits & 240 bits & 80 bits & 160 bits & (240 bits) \\
\hline
\end{tabular}

Table 1. Data size per DL/UL slot with various modulation and coding schemes.

\begin{tabular}{|c|c|c|c|c|c|c|}
\hline \multirow{2}{*}{} & \multicolumn{3}{|c|}{ DL (28 OFDM symbols) } & \multicolumn{3}{c|}{ UL (18 OFDM symbols) } \\
\cline { 2 - 7 } & QPSK & 16 QAM & 64 QAM & QPSK & 16AM & (64 QAM) \\
\hline CC 1/2 & 2,016 Mbit & 3,859 Mbit & 6,048 Mbit & 0,979 Mbit & 1,958 Mbit & (2,9 Mbit) \\
\hline CC 2/3 & $2,572 \mathrm{Mbit}$ & 5,376 Mbit & 8,064 Mbit & 1,305 Mbit & 2,611 Mbit & (3,9 Mbit) \\
\hline CC 3/4 & 3,024 Mbit & 6,048 Mbit & 9,072 Mbit & - & - & - \\
\hline CC 5/6 & $3,360 \mathrm{Mbit}$ & 6,720 Mbit & 10,08 Mbit & 1,632 Mbit & $3,264 \mathrm{Mbit}$ & (4,9 Mbit) \\
\hline
\end{tabular}

Table 2. Raw data rate in megabits per second considering a setting of 28 usable OFDM DL symbols and 18 usable OFDM UL symbols.

A broad range of combinations exists, however, most likely is a combination with robust coding (i.e. convolution code (CC) with rate 1/2) with modulation of QPSK or 16 QAM for the UL and 16 QAM or 64 QAM for the DL.

Each $5 \mathrm{~ms}$ AeroMACS frame starts with a DL Prefix which occupies one entire OFDM symbol. The Frame Control Header $(\mathrm{FCH})$ follows immediately the DL Prefix and contains information about the following DL Map. The DL Map and the UL Map are important management elements which tell the Mobile Stations (MSs) how the upcoming frame is to be used to exchange either data or management information. The mentioned elements of DL Prefix, FCH, DL Map, and UL Map appear in each DL subframe. The UL direction needs to schedule ranging opportunities for Mobile Stations in order to keep synchronized with the Base Station and in order to request bandwidth if a MS needs to do so. The remaining bandwidth may be used to transmit user data.

\subsection{Overview medium access control}

The IEEE 802.16 standard specifies a point to point and connection oriented link, i.e. each Service Data Unit (SDU) received from an interfacing higher layer is mapped to a unique and unidirectional service flow with specific quality of service (QoS) parameters. Thereby, the interfacing higher layer can be one of several different types.

The MAC common part sub-layer operates in a point to multipoint environment. The Base Station (BS) is the only user of the Downlink (DL) resources, whereas the Mobile Stations have to share the Uplink (UL) resources. All MSs are able to receive DL transmissions. Based 
on the Connection Identifier (CID) carried within the generic MAC header of each MAC PDU a MS is able to determine whether a MAC PDU is destined to it or not.

A central concept of the IEEE 802.16 standard is the usage of transport connections which allows the utilization of QoS at MAC level. Each service flow has specific QoS parameters initialized at connection setup. Thereby, different data delivery strategies can be utilized (e.g. best effort, polling, etc.).

At system initialization two pairs of management connections, namely the basic connection and the primary management connection, have to be established between the MS and the BS. A third management connection, the secondary management connection, may be established, too. However, such a connection is only mandatory for managed "subscriber stations". In certain circumstances especially if remote airport equipment is being used such a secondary management connection would probably make sense. However, the basic management connection shall be used to transmit short and time urgent MAC management messages while the primary management connection shall be used to exchange longer and delay tolerant MAC management messages.

\subsection{Convergence sub-layer options}

The convergence sub-layer (CS) in the case of the IEEE 802.16 standard specifies the interface towards higher layer protocols. The standard provides a variety of convergence sub-layer options in order to provide the possibility to interface with a versatile set of higher layer protocols. The principle functions of the convergence sub-layer are accepting and interpreting the higher layer protocol header and consequently the mapping of this information to a specific service flow. Additionally, header compression techniques or any other appropriate processing may be conducted by the convergence sub-layer protocol.

The AeroMACS draft profile foresees only the IP CS (support of IPv6), however, optionally also the Ethernet CS is supported. In principle the issue of the convergence sub-layer seems straight forward - either AeroMACS supports higher layer protocol A or higher layer protocol B. However, recalling the principle design issues of layered communication protocol architectures there may be issues as discussed below.

Figure 3 shows a generic view of a network reference model containing layers, interfaces, and protocols. Thereby, a layer of a network can be seen as an abstraction of a service or services to be provided to its higher layer. In such a way the implementation details are hidden from the service user, that is the higher layer. The higher layer accesses these services through a well defined interface (also known as service access point). The specification of clean interfaces provides the advantage to exchange completely different implementations of layers, assuming that the new implementation provides the same set of services as the old implementation did. Virtually, two communicating hosts are exchanging information on layer $n$ using a layer $n$ protocol. However, real communication takes place only via the physical transmission medium. Generally, a protocol specifies the rules and conventions to be used in a communication.

A list of protocols used by a certain system, one protocol per layer, is called a protocol stack. Services and protocols are distinct concepts, although they are frequently confused. A service is a set of primitives (operations) that a layer provides to the layer above it. The service defines what operations the layer is prepared to perform on behalf of its users, but it says nothing at all about how these operations are implemented. A service relates to an interface between two layers, with the lower layer being the service provider and the upper layer being the service user. 


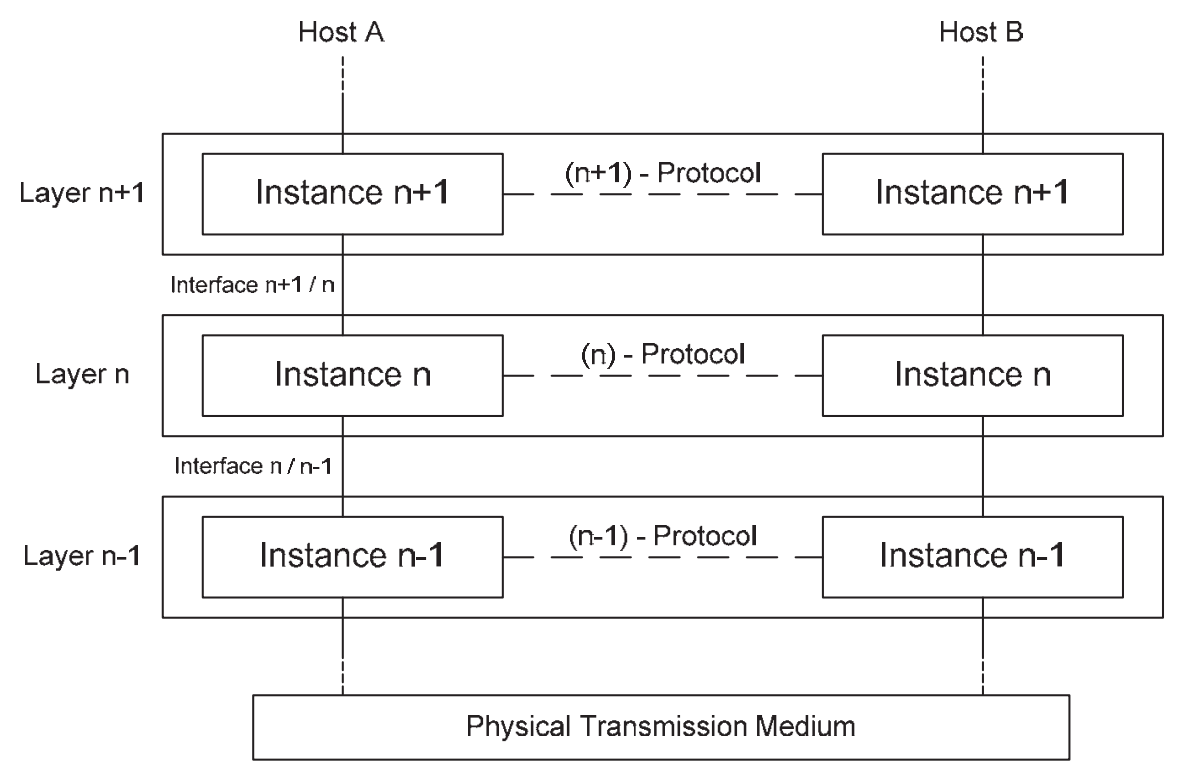

Fig. 3. A generic protocol stack.

A protocol, in contrast, is a set of rules governing the format and meaning of the frames, packets, or messages that are exchanged by the peer entities within a layer. Entities use protocols in order to implement their service definition. They are free to change their protocols at will, provided they do not change the service visible to their users. In this way, the service and the protocol are completely decoupled.

Considering the two options of the packet based convergence sub-layer, namely IP CS and Ethernet CS. The offered service differs from the IP point of view and may cause problems when considering IP over AeroMACS (c.f. Chapter 3).

\subsection{MAC PDU formats}

The IEEE 802.16 standard offers various options for fragmenting and reassembling MAC Service Data Units. Thereby, a MAC SDU may be of variable or fixed length. In the case of AeroMACS a variable length of MAC SDUs shall be allowed. Generally, a MAC Protocol Data Unit shall be of the form as depicted in Figure 4. Each MAC PDU starts with a fixed length header of 6 bytes (the generic MAC header). A MAC PDU typically contains payload and shall then be appended by a 4 bytes Cyclic Redundancy Checksum (CRC). The payload itself may further contain several sub-headers $(\mathrm{SH})$. The fragmentation sub-header is used if an entire MAC SDU does not fit into a single MAC PDU. The packing sub-header is used if several MAC SDU are packed together into a single MAC PDU. Multiple MAC PDU may also be concatenated during a single burst.

If the MAC PDU does not contain payload data MAC header needs no CRC as the MAC header itself contains a header checksum. The generic MAC header contains the connection identifier (CID) of the connection with which the PDU is associated. The MAC PDU does not contain any source or destination address in its header. The tentative AeroMACS draft profile uses the same MAC PDU format specification as the WiMAX Forum (WMF) Mobile System specification (WiMAX Forum, 2010). 


\subsection{Automatic Repeat Request (ARQ)}

Generally, Automatic Repeat Request (ARQ) protocols are used to synchronize data flows between sending and receiving entities. Thereby, the flow control procedure takes care that the data source is not overloading the data sink. Also erroneous data packets are indicated to the source (through negative acknowledgments).

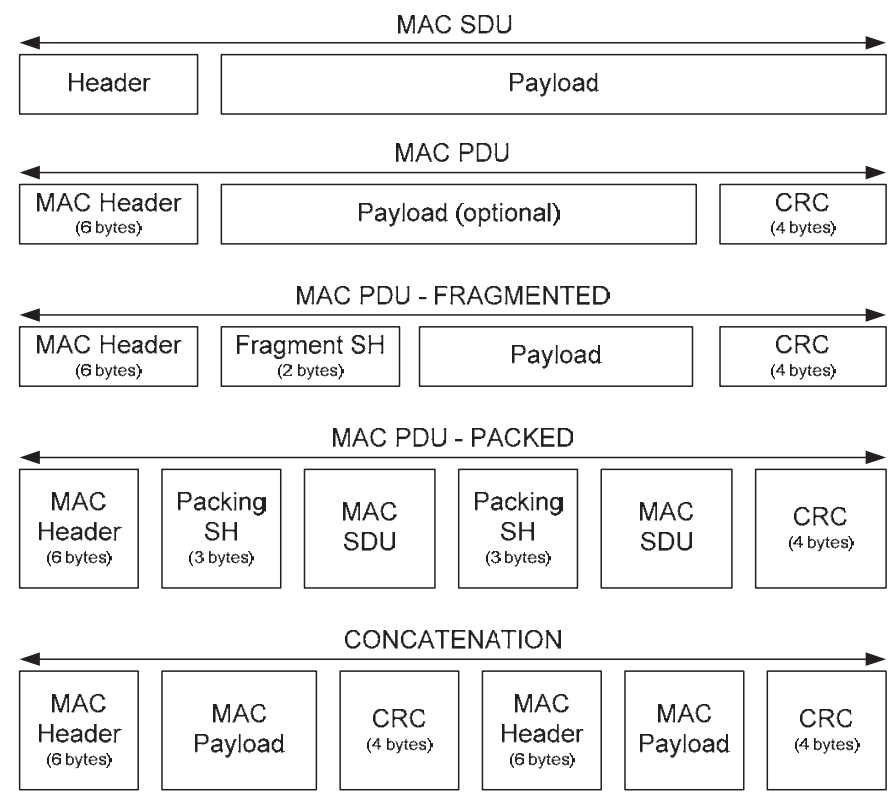

Fig. 4. Overview of different MAC PDU options.

The IEEE 802.16 standard offers four different types of ARQ, namely, go-back-n, selectivereject, and two combinations of go-back-n and selective-reject. Go-back-n may also be called as cumulative ARQ. An ARQ information element has at least a size of 4 bytes and at most of 12 bytes. The basic components of an ARQ information element are the connection identifier field and the block sequence number (BSN) field. The CID identifies the transport connection and the BSN is differently used dependent on the ARQ type.

The ARQ protocol of the IEEE 802.16 standard is based on ARQ blocks, which all have a size of ARQ_BLOCK_SIZE in bytes. An exception may only be for the last ARQ block of an SDU which may be smaller. Each incoming SDU from a higher layer is logically divided into a number of ARQ blocks. Thereby, each ARQ block gets a BSN. Compare Figure 5 which is showing an example with ARQ_BLOCK_SIZE set to 32 bytes and a sequence of three arriving SDU with a size of 90, 10, and 64 bytes.

\begin{tabular}{|c|c|c|c|c|c|}
\hline \multicolumn{3}{|c|}{$\begin{array}{c}\text { SDU } 1 \\
\text { (90 bytes) }\end{array}$} & $\begin{array}{c}\text { SDU } 2 \\
\text { (10 bytes) }\end{array}$ & \multicolumn{2}{|c|}{$\begin{array}{c}\text { SDU } 3 \\
\text { (64 bytes) }\end{array}$} \\
\hline $\begin{array}{c}\text { block } 1 \\
\text { (32 bytes) }\end{array}$ & $\begin{array}{c}\text { block } 2 \\
\text { (32 bytes) }\end{array}$ & $\begin{array}{c}\text { block } 3 \\
\text { (26 bytes) }\end{array}$ & $\begin{array}{c}\text { block } 4 \\
\text { (10 bytes) }\end{array}$ & $\begin{array}{c}\text { block } 5 \\
\text { ( } 32 \text { bytes) }\end{array}$ & $\begin{array}{c}\text { block } 6 \\
\text { ( } 32 \text { bytes) }\end{array}$ \\
\hline
\end{tabular}

Fig. 5. Example of different SDU with an ARQ block size of 32 bytes. 
The single blocks may be packed into one or several MAC PDUs. Using the above example all 6 ARQ blocks could be packed together to a single MAC PDU, however, the ARQ blocks could also be packed into 3 separate MAC PDU where each MAC PDU carries 2 ARQ blocks. ARQ blocks from the same SDU with consecutive block sequence numbers can be grouped together into an SDU fragment. Each fragment (or single ARQ block) is preceded by a packing sub-header (PSH) which carries the BSN of the first ARQ block and the length of the fragment in bytes. This allows the receiver to decode the ARQ blocks again. If the fragment length is not a multiple of ARQ_BLOCK_SIZE, it means the last block in the fragment is smaller. The complete MAC PDU for the example case above where all ARQ blocks are sent in a single MAC PDU could look like as depicted in Figure 6.

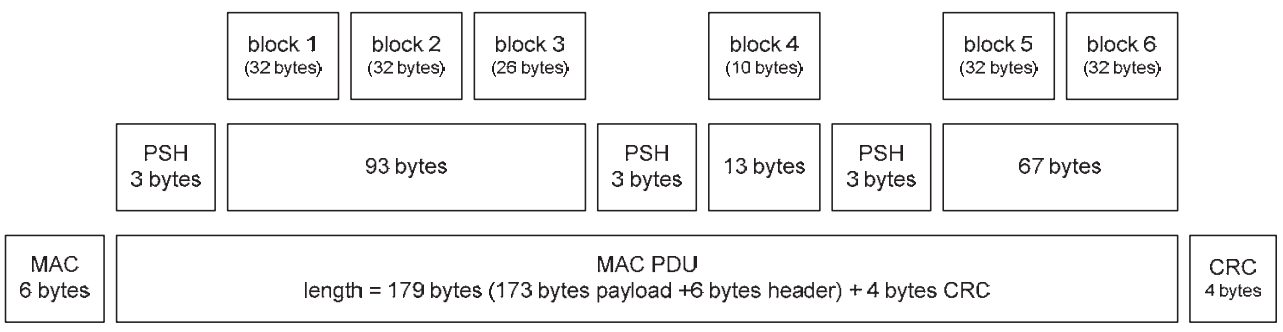

Fig. 6. Example MAC PDU - MAC packing.

Figure 6 continues the example from above where 3 MAC SDUs are packed into a single MAC PDU. Each MAC SDU (fragment) is prefixed by a PSH which has a length of 3 bytes. Additionally, the MAC PDU overhead accounts for 10 bytes - i.e. the generic MAC header with 6 bytes and the CRC with 4 bytes. This example of packing MAC SDU results in an overhead of 19 bytes for a payload of 164 bytes.

\subsubsection{ARQ acknowledgment types}

Acknowledgment type 0 (i.e. selective ACK entry) contains up to 4 fixed length acknowledgment maps. The length of such a map is 16 bits where each bit indicates whether a corresponding ARQ block has been received successfully (i.e. bit is set) or not (i.e. bit is not set). When using the selective ACK entry option the BSN corresponds to the first bit of the following acknowledgement map. It is important to realize that such an acknowledgement type is only applicable if more than or equal to 16 ARQ blocks have been received without prior sent acknowledgment.

Acknowledgment type 1 (i.e. cumulative ACK entry) uses the BSN to cumulatively acknowledge all ARQ blocks received. This acknowledgment type has a fixed size of 4 bytes. Acknowledgment type 2 (i.e. cumulative with selective ACK entry) is a combination of acknowledgment type 0 and type 1 . In this case the BSN is interpreted as cumulative acknowledgment and the first bit of the following map is set - the remaining bits of the map can be used as in type 0 .

Acknowledgement type 3 (i.e. cumulative with block sequence ACK entry) is a combination of type 1 and a series of sequence ACK maps. The BSN acknowledges all correctly received ARQ blocks cumulatively. The sequence ACK map contains either two sequences with a length given in 6 bits or three sequences with a length given in 4 bits. Thereby, each sequence specifies a number of consecutive BSN entries, with the first sequence starting at 
the cumulative BSN plus one (which is always a negative acknowledgment; otherwise the cumulative BSN would be increased).

Figure 7 shows a hypothetical example of 32 contiguous ARQ blocks where some of them were received correctly and some of them were received erroneously. Erroneous blocks are marked with an X. The differences of the various acknowledgment types become obvious when applying each acknowledgment type separately to the same set of ARQ blocks. In this case type 0 (i.e. selective ACK entry) is capable to give feedback on every received ARQ block. The reason therefore is that the number of ARQ blocks fits exactly two acknowledgment maps of 16 bits. The type 1 acknowledgment (i.e. cumulative ACK entry) can only confirm the receipt of the first three correctly received ARQ blocks. The type 2 acknowledgment (i.e. cumulative and selective ACK entry) is capable of acknowledging only the first 18 ARQ blocks. The reason therefore is that selective ACK map sizes are fixed to a length of 16 bits, the remaining 14 ARQ blocks cannot be acknowledged by this method until erroneously received ARQ blocks are being retransmitted and received correctly. The type 3 acknowledgment (i.e. cumulative with block sequence ACK entry) uses sequences to acknowledge sequences of correctly or erroneously received ARQ blocks. If the option is used with 2 block sequences per sequence map (3a) only 20 ARQ blocks can be acknowledged. Using the option with 3 block sequences per sequence map (3b) acknowledges 27 ARQ blocks in this case. This example does not work well for the block sequence map option as the sequences are very short.

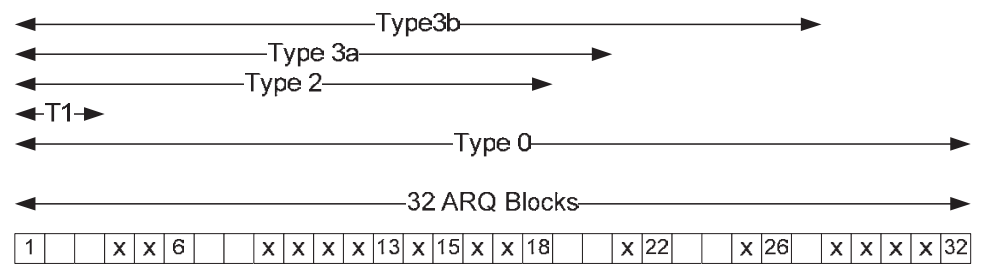

Type 0: Selective ACK (size 8 bytes) BSN: 1 MAP: 1110011100001010 MAP: 0111011101100001

Type 1: Cumulative ACK (size 4 bytes) BSN: 3

Type 2: Cumulative + Selective ACK (size 6 bytes) BSN: 3 MAP: 1001110000101001

Type 3a: Cumulative + Sequence ACK (size 12 bytes) -2 sequence maps

\begin{tabular}{|l|l|l|l|l|l|}
\hline BSN: 3 & SEQ: 01 & 3 & SEQ:01 & 4 \\
\hline
\end{tabular}

\begin{tabular}{l|l|l|l|l|l|}
\hline SEQ: 01 & 1 & 1 & SEQ: 01 & 2 & 3 \\
\hline
\end{tabular}

Type 3b: Cumulative + Sequence ACK (size 12 bytes) -3 sequence maps

\begin{tabular}{|c|c|c|c|c|c|c|c|c|}
\hline BSN: 3 & SEQ: 010 & 2 & 3 & 4 & SEQ: 101 & 1 & 1 & 1 \\
\hline & SEQ: 010 & 2 & 3 & 1 & SEQ: 101 & 3 & 1 & 2 \\
\hline
\end{tabular}

Fig. 7. Illustration of the capabilities of the different ARQ acknowledgment types. 
This example illustrates the functionality of the different ARQ options which may be used by the AeroMACS profile. Each ARQ option has its advantages depending on the frequency acknowledgments are sent (e.g. after the receipt of each MAC PDU, or after a certain amount of time, etc.), the pattern of the occurred errors, or the computational complexity.

The WiMAX Forum ${ }^{\mathrm{TM}}$ Mobile System Specification requires ARQ acknowledgment types 1, type 2, and type 3 to be implemented. ARQ acknowledgment type 0 is optional. The AeroMACS profile intends to support the same set of acknowledgment options.

Each acknowledgment type has its advantages but is dependent on feedback intervals, error patterns, and computational complexity. The size of the ARQ block has an impact as well, if large ARQ blocks are used it is more unlikely to fill acknowledgment maps or acknowledgment sequence maps. The standard does not specify any strategy how and when ARQ acknowledgments shall be scheduled.

\subsection{Qualtiy of Service (QoS)}

Quality of Service in IEEE 802.16 is supported through the concept of unicast transport connections. These transport connections are called service flows, where each service flow utilizes a particular set of QoS parameters. The standard provides several QoS parameters to be adjusted; for instance maximum sustained traffic rate, maximum traffic burst, minimum reserved traffic rate, maximum latency, etc. - in principle latency, jitter, and throughput assurance.

Service flows are either provisioned or dynamically added by the Base Station or optionally by the Mobile Station. How to provision service flows is out of the scope of the IEEE 802.16 standard, consequently it is also not specified in the AeroMACS draft profile. Certain service flows may be added dynamically for instance after the network entry procedure. The standard provides options to create, change, and delete a service flow dynamically. Such a procedures can be either initiated by the Base Station or by the Mobile Station. The WiMAX mobile profile makes these options mandatory to be supported by the Base Station. The capability to dynamically create or change a service flow is optional for a Mobile Station, however, the deletion of a service flow is mandatory. The AeroMACS profile intends to support only the dynamic service flow creation, change, and deletion procedures to be initiated by the Base Station.

How these service flows are initiated and / or triggered is not specified by the AeroMACS profile. QoS parameters of ATC traffic flows shall probably be regulated while QoS parameters of AOC traffic flows may be provider dependent.

\subsection{Scheduling \& data delivery services}

There are different possibilities to provide bandwidth to a Mobile Station, realized through a scheduling service. Uplink request and grant scheduling is performed by the Base Station in order to provide each Mobile Station with bandwidth for uplink transmissions or opportunities to request bandwidth. By specifying a scheduling type and its associated QoS parameters, the Base Station scheduler can anticipate the throughput and latency needs for the uplink traffic and provide polls and/or grants at the appropriate times. The different scheduling services are:

- Unsolicited Grant Service (UGS)

- $\quad$ real-time Polling Service (rtPS)

- $\quad$ non-real-time Polling Service (nrtPS) 
- $\quad$ Best Effort (BE)

The unsolicited grant service (UGS) is intended for real-time applications which generate fixed-rate data. Among others QoS parameters such as tolerated jitter, minimum reserved traffic rate, maximum latency, and the unsolicited grant interval are defined. This means that a service flow with a data delivery service of UGS gets periodically UL resources assigned without requesting them each time.

The real-time Polling service (rtPS) is intended for real time applications with variable bit rates. Among others QoS parameters such as maximum latency, minimum reserved traffic rate, traffic priority, and the polling interval are defined. In this case the resource scheduler polls a Mobile Station regularly at fixed intervals. These polls may be used to request bandwidth.

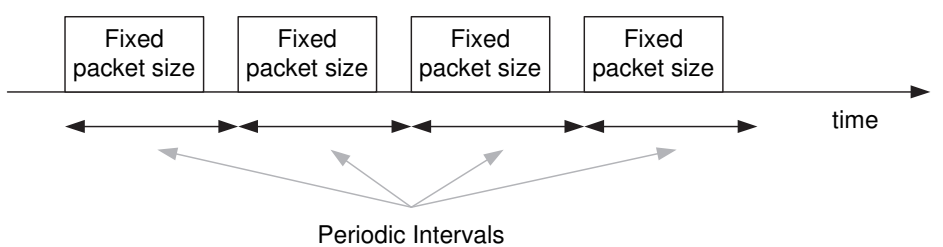

Fig. 8. The Unsolicited Grant Service (UGS) usable for uplink transmissions.

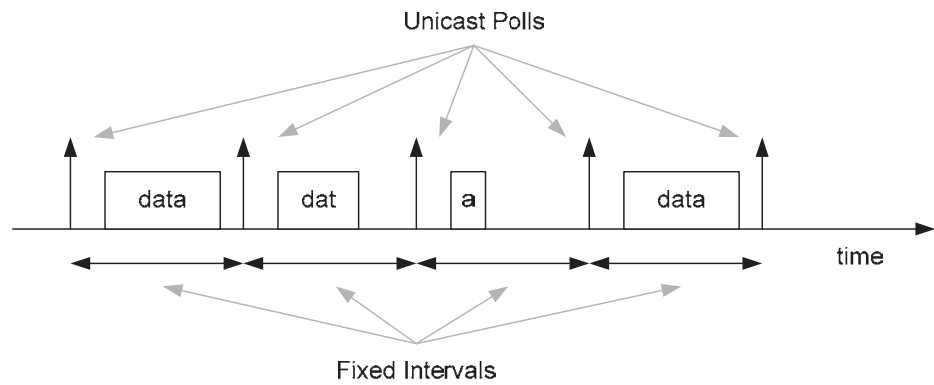

Fig. 9. The real-time Polling service (rtPS) usable for uplink transmissions.

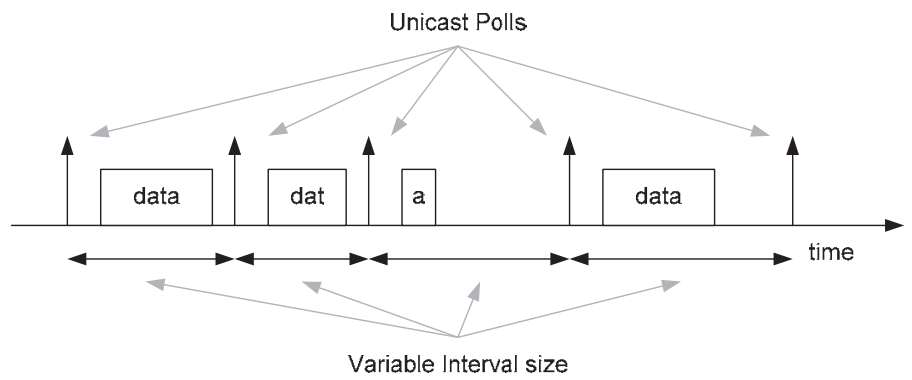

Fig. 10. The non-real-time Polling Service (nrtPS) usable for uplink transmissions. 
The non-real-time Polling Service (nrtPS) is intended for applications which require guaranteed data rate but are insensitive to delays. QoS parameters such as minimum reserved traffic rate, maximum sustained traffic rate, and traffic priority are defined. In this case the unicast polls are issued at a variable interval length (dependent on the available resources). The polls may be used to request bandwidth.

The Best Effort (BE) service is intended for applications with no rate or delay requirements. In this case bandwidth request ranging opportunities are provided to transmit bandwidth request ranging codes. If a bandwidth request range code is successfully received by a Base Station it polls the associated Mobile Station.

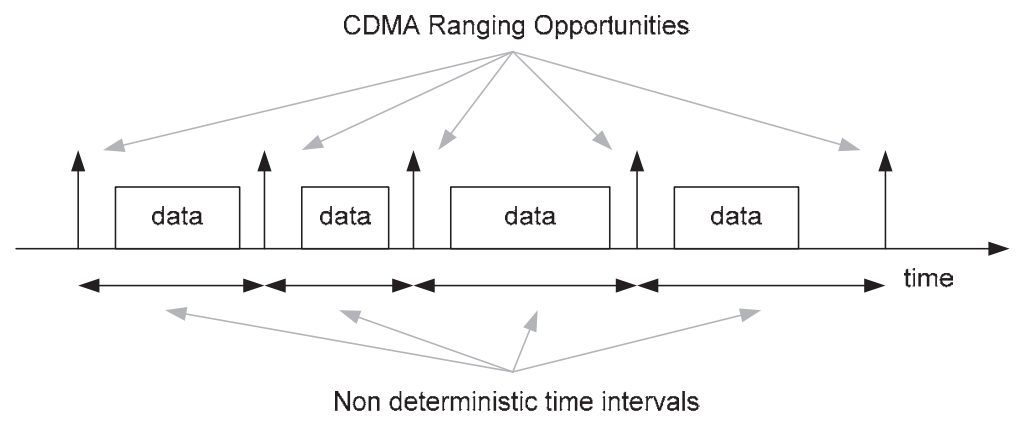

Fig. 11. The Best Effort (BE) service usable for uplink transmissions.

For the downlink direction similar QoS classes can be utilized. However, these are called slightly different but have comparable QoS parameters. The scheduler does not need to consider any polls or ranging opportunities for the downlink, though. The different QoS classes or data delivery services are:

- Unsolicited Grant Service (UGS)

- $\quad$ Real-Time Variable Rate (RT-VR) service

- Non-Real-Time Variable Rate (NRT-VR) service

- $\quad$ Best Effort (BE) service

What kind of QoS class a specific application or set of applications will require is dependent on the requirements. How the different data delivery services and scheduling strategies are implemented is not specified by the standard. Thus, they are vendor dependent. In any case the communication service provider has to assure that safety related communication is preferred over non safety related communication. It might be that a simple priority scheme with a best effort service is sufficient.

\subsection{Request-grant mechanisms}

A Mobile Station is required to support at least three different connections. That is, two management connections which are set up at network entry and one data bearer to transmit user data.

Every connection with a QoS service other than UGS needs to adapt its resource requirements. This is done through bandwidth requests. This is a mechanism where a Mobile Station indicates to the Base Station that it requires uplink resources. Bandwidth requests are either sent as standalone Bandwidth Request (BR) headers or as a Piggy Back Request (i.e. included in the Grant Management Sub-header (GMSH)). 
Bandwidth Requests may be either incremental or aggregated. When a BS receives an incremental BR, it shall add the quantity of bandwidth requested to its current perception of the bandwidth needs of the connection. When the BS receives an aggregate BR, it shall replace its perception of the bandwidth needs of the connection with the quantity of bandwidth requests. Piggybacked bandwidth requests are always incremental.

The Base Station issues resource grants towards a Mobile Station based on the basic CID (i.e. basic management connection). This means that a Mobile Station is able to utilize the concept of bandwidth stealing where a certain amount of requested bandwidth for a specific QoS class may be utilized differently. However, the resource requests are based on the transport connection which requires bandwidth. If a Base Station polls a Mobile Station it typically assigns enough resources to issue a bandwidth request.

\section{IPv6 over AeroMACS}

The Network Working Group (NWG) of the WiMAX Forum ${ }^{\mathrm{TM}}$ has defined a network architecture for IEEE 802.16 sub-networks. Thereby, considering topics at layers above those defined by the 802 standards. The Internet Engineering Task Force (IETF) has worked out a Request For Comment (RFC) "Transmission of IPv6 via the IPv6 Convergence Sub-layer over IEEE 802.16 Networks" (RFC 5121, 2008) which provides a full conformant IPv6 connectivity through an IP point to point link. This solution fits the general business use case where each subscriber resides in its own sub-network. However, the requirements of the sub-network in an aeronautical environment might be different than the one of an ordinary business use case. Running IPv6 over AeroMACS shall be fully compliant to the IP standard, thereby, IP multicast shall be supported preferably in an efficient manner. It might also be desirable to support multicast at link layer which is difficult with point to point links. The problem statement and possible different solutions are discussed in the following. First of all it is important to identify the relevant concepts of IPv6 addressing. In IPv6, nodes are attached to an access network via an interface, which is given at least one IPv6 address (i.e. the link local unicast address). Within this context a node can be understood as a device which implements IPv6. This means that an interface gets one or more IPv6 addresses assigned and not the node itself, which is a fundamental concept of IP. In other words a node may host several network interfaces which have different addresses. Thereby, the same node may be reachable through different IPv6 addresses.

An IPv6 capable node must be able of configuring its IPv6 address autonomously. An IPv6 address is created through a valid interface identifier and a valid subnet prefix. The subnet prefix may be a constant link local prefix (i.e. FE80::0), an advertised prefix received by Router Advertisements, or a prefix by a DHCPv6 server. The prefix is only valid on the link on which it is received - the prefix shall not be used on different links. Link local addresses allow communications between devices on a local link; such addresses cannot be used to communicate outside a network link.

Native multicast capability can be described through the following general concepts of the IP addressing model - first through the concept of a link and secondly through the concept of a subnet. A link is a term used to refer to a topological area bounded by routers that decrement the IPv6 Hop Limit when forwarding a packet (c.f. RFC 4903, 2007). The term subnet is generally used to refer to a topological area that uses the same address prefix, where that prefix is not further subdivided except into individual addresses (c.f. RFC 4903, 2007). Thereby, it is important to 
recognize that IPv6 continues the IPv4 model that a subnet is associated with one link. Multiple subnets may be assigned to the same link (c.f. RFC 4291, 2006). Ideally, the Data Link layer addressing mechanisms can be directly used for the Internet Protocol addressing method. Some of the Internet layer protocols (e.g. Address Auto-configuration (RFC 4862, 2007), Neighbour Discovery (RFC 4861, 2007), Dynamic Host Configuration Protocol (RFC 315, 2003), or more generally protocols used for service discovery or name resolution) require native multicast capability of the underlying link, that is data packets can be distributed to all interested nodes on the same link without a decrement of the IPv6 Hop Limit field. If such a native multicast capability is not given by a certain link technology, an IP link model has to be presented towards the Internet layer which fulfils this requirement.

In principle, if a physical link characteristic is problematic at the Internet layer, mechanisms have to be defined that the link model appears properly at the Internet layer. The Internet Architecture Board (IAB) recommends using one of the two following models: The multiaccess link model or the point to point link model. These models, if implemented properly, have no problems regarding the IP addressing model and the native multicast capability (c.f. RFC 4903, 2007).

\subsection{IP point-to-point link model}

Figure 12 considers a generic configuration of the IP point to point link model. Here exactly two nodes (i.e. Node A and Node B) are located on the same link. Native multicast is supported, that is, both nodes on the link are able to receive data packets which are sent to a link local multicast address. Also, both nodes can communicate with each other without any IPv6 Hop Limit decrement. Any Layer 2 device (e.g. bridges, switches, etc.) connected in the middle of the link is allowed. In terms of AeroMACS Node A would reflect the Mobile Station (MS) and Node B would be an Access Router, respectively.

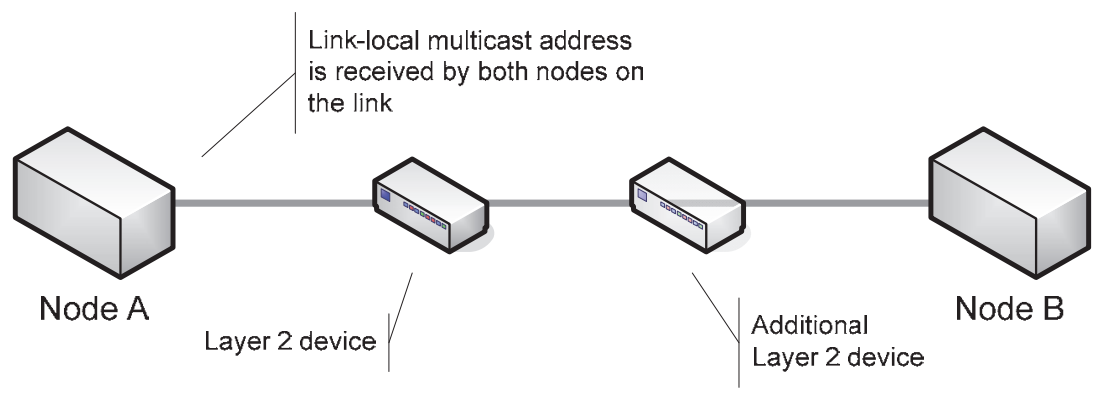

Fig. 12. A generic configuration of a "Point-to-Point Link Model".

\subsubsection{Single prefix per mobile station}

Considering the IP point to point link model presented to the network layer one possibility is to provide a single prefix to each Mobile Station. The AeroMACS communication system may make use of the IP convergence sub-layer (CS) or the Ethernet convergence sub-layer. In principle both solutions would work for the point to point connection, however, from a standard's point of view the two components are distinguished as the Ethernet configuration would allow a bridging functionality which the IP configuration does not.

Examining the case where data traffic is transmitted from the Mobile Station to the access router the following steps occur (cf. Figure 13): 
1. An IPv6 packet is handed over to the convergence sub-layer. There the protocol fields of the higher layer data packet map to a Service Flow Identifier (SFID) that further maps to a Connection Identifier (CID). The connection identified through the CID is used to transmit the higher layer data packet to the Base Station (BS) - which is a layer two device as depicted in the Figure 12.

2. The "Data Path Function" of the BS encapsulates the IPv6 packet into a Generic Routing Encapsulation (GRE) tunnel. A unique GRE tag maps to the SFID of the connection.

3. The "Data Path Function" of the ASN Gateway receives the IPv6 packet and forwards it accordingly.

The other direction works analog. The "Data Path Function" of the ASN GW and the BS use the unique GRE tag to map to the SFID and CID of a connection, respectively. In such a way a virtual tunnel from the Access Router to the Mobile Station and vice versa is created. The "Data Path Function" creates the tunnel on a per service flow granularity. From an IP point of view these GRE tunnels have to be presented to the IP layer as "virtual interfaces" as each interface is more or less a single link. In such a way distinct prefixes have to be advertised on each link making the link a pure point to point link at layer 2 and layer 3 . The main drawback of that solution is that standard multicast capabilities of the AeroMACS subnetwork are disabled by default.

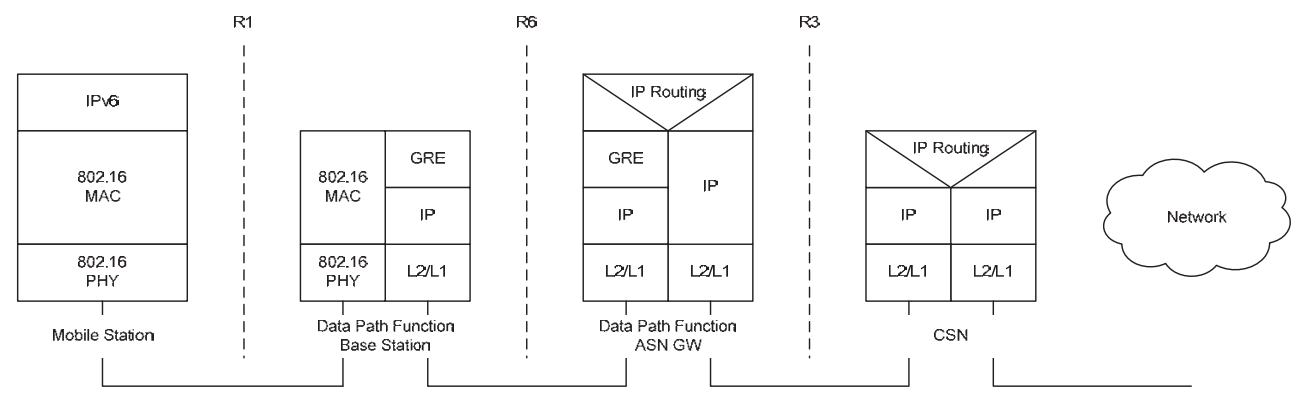

Fig. 13. Protocol stacks of different entities, considering an IP point to point link model.

\subsubsection{Shared prefix for all mobile stations}

If a shared prefix is used for all "Virtual Interfaces" the IP link model is violated, as the same prefix is advertised on different links. If only a single IP prefix is used (and native multicast among all attached Mobile Stations is being supported) an additional function is required (some kind of backend process). If a shared prefix is being needed for an AeroMACS system, the AeroMACS link as such has to be presented as a single link to the IP process. Therefore, some kind of backend process (or a similar approach like MLD snooping) is required. Such a backend process is no standard solution and would require a separate specification and implementation - such a solution is probably not desired.

\subsection{IP multi-access link model}

Figure 14 shows a generic configuration of a multi-access link model. One link is shared by one or more nodes (i.e. Node A, Node B, Node X, etc.). Thereby, the link may be attached to one or more routers. However, a router is not stringently necessary. Native multicast is supported, that is, all interested nodes on the link are able to receive data packets which are sent to a link local multicast address. Additionally, two nodes on the same link can 
communicate with each other without any IPv6 Hop Limit decrement. Any Layer 2 device (e.g. bridges, switches, etc.) connected in the middle of the link is allowed.

The IEEE 802.16 over Ethernet option would provide such functionality - realized through the Ethernet CS interface and an Ethernet bridge (realized as layer 2 device). The AeroMACS link is presented to the IPv6 capable router only as a single interface. Note that the presentation of the single interface to the IP process as such has to be handled by the "Data Path Function" similar to the "backend process" presented earlier. The "Data Path Function" will encapsulate the data packets into a GRE tunnel and map them accordingly to a SFID and CID, respectively. Such a solution would be only possible if the Ethernet CS option is being used as the IP CS solution does not offer any possibility to bridge the data traffic.

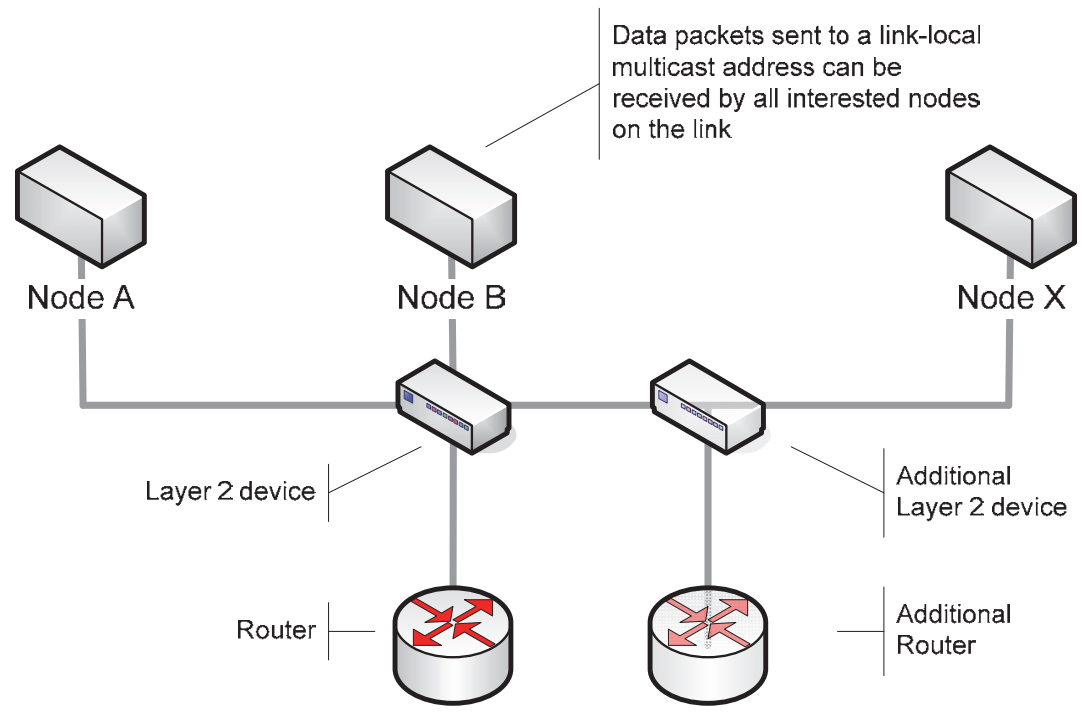

Fig. 14. A generic configuration of a "Multi-Access Link Model".

Alternatively the general encapsulation convergence sub-layer could be used to come up with a specific solution for the aviation case. However, this is no standard solution and would require additional development resources. A process which is not desired.

\subsection{IP multicast}

If one of the above mentioned IP models is implemented properly for the AeroMACS communication system IP multicast is always possible, as the system is fully IPv6 compliant. However, the advantage of IP multicast should be to transfer the data packet only as often 
as necessary. Utilizing an IP point to point link model means that each multicast data packet transmitted to a group of multicast listeners belonging to the same cell and / or sector of a Base Station needs to be multiplied by the amount of listeners. Utilizing the Multi-Access link model means that the data packet is transmitted only once to the same group of multicast listeners belonging to the same cell/sector of a Base Station - this might increase the efficiency of an AeroMACS communication system.

In standard business cases of the mobile communication industry this may have no large impact. However, the aeronautical business case may have a larger interest in multicast applications. Therefore, the advantages and drawbacks of the different solutions shall be analyzed in depth in the future.

\section{Future data traffic}

Different types of application may utilize the AeroMACS communication system, among these could be fixed users, mobile users, and aircraft. By the term fixed users airport LAN extension could be considered such as unique equipment (terminals, cameras, etc.). Also Aeronautical Navigation Service Provider (ANSP) managed equipment like area navigation (RNAV) systems could be interpreted as fixed user. Mobile users would be airport surface vehicles such as airport trucks (catering, maintenance, refueling trucks, etc.) or mobile terminals which support for instance voice over IP. Aircraft would utilize an AeroMACS system by applications such as Air Traffic Control (ATC), Aeronautical Operational Communications (AOC), or Aeronautical Administrative Communications (AAC) which may have a direct operational and safety impact.

In order to assess the performance of an AeroMACS communication system properly an assumption on the data traffic load for airport surface communications was necessary. This sub-chapter summarizes the results of the data traffic load analysis for aircraft and mobile users conducted during the course of the SANDRA project (SANDRA). Services as anticipated by the COCRv2 report (COCR, 2007) and the AOC data link dimensioning report (AOC, 2010) were considered. In order to simulate a data traffic model the following inputs were necessary:

- An air traffic model: A simulation of the aircraft movement on the airport. That is, departing aircraft moving from ramp to runway, arriving aircraft moving from runway to ramp, and ground vehicles.

- Supported applications: Data link applications envisaged for the airport domain and their trigger events. Note that most aeronautical data link applications are triggered by events related to the progress of the flight. The events are provided by the air traffic model.

- Scenarios: These are the different use cases of the airport data link. This relates mostly to the amount of aircraft serviced, and the various sets of supported applications.

The output of the data traffic model is the statistical description of the expected offered load at application layer (ISO/OSI layer 7). The offered load is the amount of data produced by the applications - this does not include any overhead of the transport layer, network layer, or data link layer. Details about the implemented model can be found in (Ehammer, 2011). The outcome of the evaluation showed that ATC applications contribute insignificantly to the offered load (in the order of a couple kbits/second), as these applications are mostly short. However, certain AOC applications may contribute significantly to the overall load, especially those related to software updates or post flight procedures. Results showed that 
an average offered load of several megabits per second is possible. Thus justifying an introduction of a broadband wireless communication system at airports.

\section{Simulation results}

Within the SANDRA project MAC performance simulations have been conducted in 2011. Initial results are presented within this sub-chapter. In principle there are two different sets of WiMAX functions defined by the Mobile System Profile (as of IEEE 802.16-2009) - i.e. a set of functions to establish point to point connections over which data can be exchanged and a set of functions to support mobility. Within this sub-chapter a performance evaluation regarding data exchange functions is given.

In order to perform the evaluation a tool chain as depicted below has been used. Thereby, the parameter set is defined through simulation scenarios. The simulation itself is built according to requirements defined by simulation scenarios. The statistic program uses the XML based output data produced by the simulation as input to generate HTML reports which shall contain all necessary statistics in order to assess the different functions of the AeroMACS MAC layer. A similar approach has been conducted in several projects using the method described in (Ehammer, 2008).

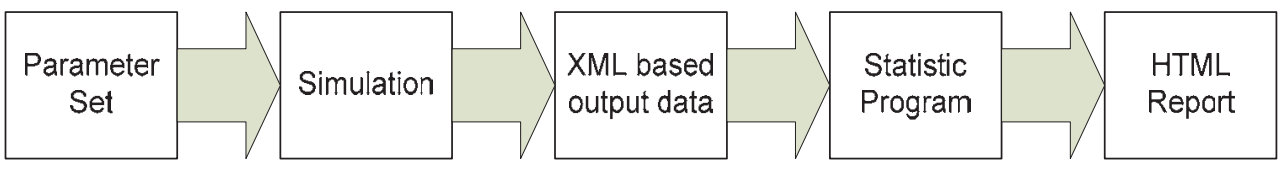

Fig. 15. Simulation tool chain.

The simulation effort concentrated solely on the MAC layer as depicted in Figure 16 below. The data transported through the connection (Mobile Station to Base Station) has been elaborated in a separate task (c.f. Chapter 3). However, the data traffic used for this evaluation is a synthetic data stream using a constant bit rate. The Physical Layer is modeled very simple through a uniformly distributed bit error rate (BER).

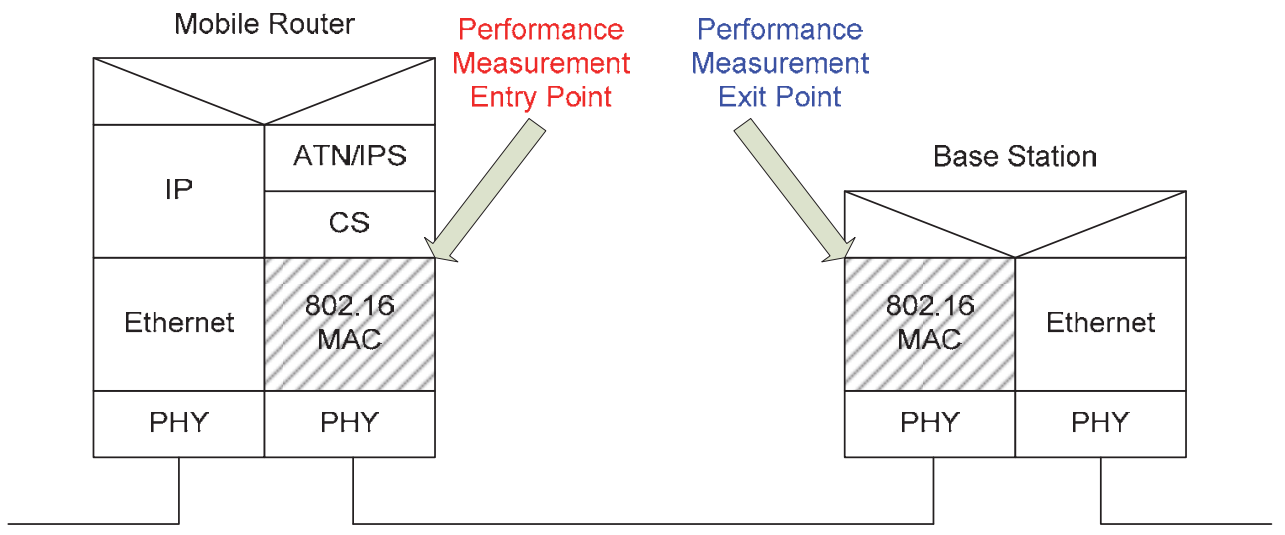

Fig. 16. Protocol stack of involved entities. 


\subsection{Evaluation}

A basic set of parameter settings is evaluated against

- A varying bit error rate (the BER scenario).

- A varying load (the LOAD scenario)

- A varying amount of active users within the cell (the PIAC scenario).

Thereby, parameters such as latency, throughput, or loss are measured. Detailed evaluations show overhead figures resulted from the protocol itself and overhead caused through retransmissions due to bad channel conditions. The evaluation scenario of varying bit error rates assumes a constant load and a constant amount of active users. Similarly, the evaluation scenario of varying load assumes a constant bit error rate and a constant amount of active users. Finally, the evaluation scenario of varying active users assumes a constant bit error rate and a constant load. For each evaluation a scenario is simulated several times in order to gain appropriate confidence intervals.

\subsubsection{Reference scenario}

The reference simulation scenario demonstrates basic data exchange capabilities of the AeroMACS protocol. The basic set of options necessary are the fragmentation and reassembly options, the ARQ implementation with all allowed acknowledgment types set, a dynamic service flow addition capability (initiated by the Base Station after network entry), and the basic resource request and resource grant options.

The basic idea is to establish a data connection which has a QoS of "Best Effort". Additionally, this data connection shall support ARQ. Almost every time an aircraft has to transmit a data packet a resource request has to be issued - this is realized through the transmission of a CDMA ranging code via the ranging slot dedicated for periodic or bandwidth request ranging. If an aircraft has resources available it can also transmit a bandwidth request piggybacked via the "Grant Management Sub-header". Depending on the bit error rate, there will be loss. The sole bandwidth request header carries always aggregated bandwidth requests while the piggybacked bandwidth request carries incremented bandwidth requests. The maximum allowed PDU size is critical, especially if the bit error rate is high. It has to be coordinated with the ARQ block size as well. Obviously, larger ARQ block sizes than maximum fragment sizes do not make much sense.

The evaluation is based on a generic traffic generation where higher layer data packets have a typical IPv6 MTU size (i.e. 1500 bytes). The load per aircraft for uplink direction and downlink direction is assumed to be constant (evaluation in discrete steps from low load to high load). The amount of active participants (i.e. Mobile Stations or surface vehicles) per cell is assumed to be constant (evaluation in discrete steps from low to high). The bit error rate is assumed to be constant (evaluation in discrete steps, from good channel to bad channel conditions).

\subsubsection{Simulation parameter settings}

The simulation itself has a series of parameters to be considered the most important parameters for the BER evaluation scenario are briefly discussed. For the BER evaluation scenario 20 Mobile Stations are considered. Thereby, each MS shall generate on average a load of $60 \mathrm{kbps}$ for the downlink direction and a load of $30 \mathrm{kbps}$ for the uplink direction. The QoS parameters are set to Best Effort (BE). That means bandwidth request ranging opportunities have to be utilized if no resources are available when higher layer data 
packets arrive. The maximum fragment size is set to 612 bytes. The ARQ settings are 128 bytes ARQ block size, $500 \mathrm{~ms}$ ARQ retry timeout, 10 seconds ARQ block lifetime, and all allowed acknowledgment types are enabled. Furthermore, acknowledgments may be piggybacked, bandwidth resource requests may be issued via the GSMH header (piggybacked), the compressed map feature is enabled, and packing of different MAC SDU is allowed. The BER values vary from $10^{-7}$ to $10^{-3}$. The modulation and coding scheme has been chosen to be QPSK and CC rate 1/2. Different coding and modulation schemes should produce similar results except with higher throughput rates. Figure 17 shows an AeroMACS frame how it has been utilized for the presented simulation results. The DL-Prefix is present in each DL sub-frame as well as the DL and UL map. Downlink and Uplink Channel Descriptors (DCD/UCD) are transmitted periodically. Ranging opportunities such as initial and handover ranging slots or bandwidth request and periodic ranging slots are offered periodically as well. Dependent on the amount of BR ranging codes issued a variable amount of CDMA allocations have to be made available for Mobile Stations in the UL direction.

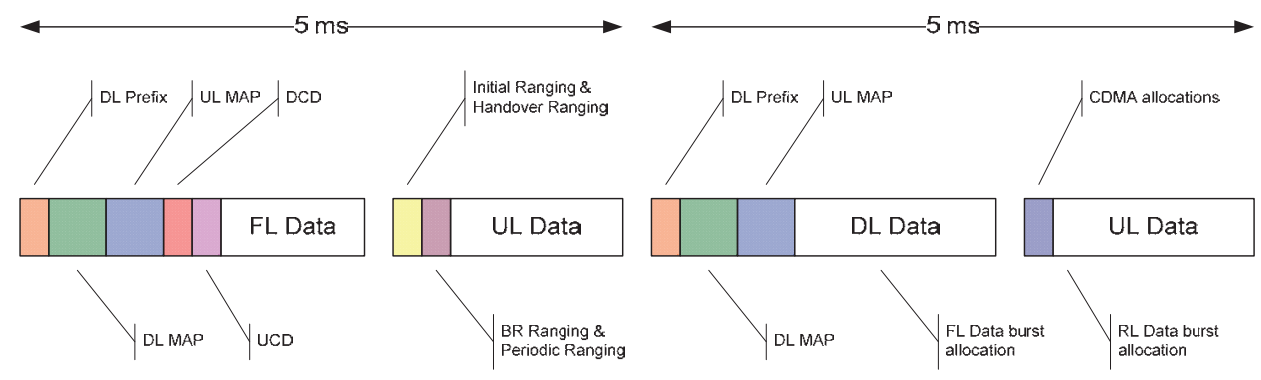

Fig. 17. Typical AeroMACS frame structure for this evaluation scenario.

\subsubsection{Selected results}

The figure below illustrate the higher layer (HiL) goodput and the data link layer (DLL) goodput. Goodput can be interpreted as user throughput, i.e. the number of useful bits per unit of time successfully forwarded by the network from a certain source address to a certain destination, excluding protocol overhead, and excluding retransmitted data packets. The figures underneath show the Forward Link (FL) and Reverse Link (RL) goodput. FL and $\mathrm{RL}$ are aeronautical terms where FL is the equivalent of DL and RL of UL, respectively. Furthermore, the figures underneath also show the FL and RL average latency for higher layer data packets and data link layer data packets. Note that the latency of data link layer packets is negligible as the transfer of a single MAC PDU takes at most $2 \mathrm{~ms}$.

The FL results (i.e. Base Station to Mobile Station) show a controlled behavior until a BER of $10^{-5}$. The higher layer data packets are still delivered at a BER of $5 \times 10^{-5}$, however the DLLgoodput increases due to re-transmissions caused by ARQ timeouts. As a result the average latency increases from approximately $100 \mathrm{~ms}$ to $1000 \mathrm{~ms}$. Further decreasing the the channel (i.e. BER equals 10-4) results in massive loss of higher layer data packets. packets. Higher layer packets are dropped as soon as the ARQ block lifetime of a MAC SDU (i.e. the higher layer data packet) expires. This is also reflected in the average FL latency which is 
slightly underneath the ARQ block lifetime of 10 seconds (the graph accounts only for higher data layer packets which were delivered successfully). Decreasing the channel quality another time (i.e. BER equals $5 \times 10^{-4}$ ) results in total loss of data as well as almost no throughput of DLL data.

Considering the RL (i.e. Mobile Station to Base Station) a controlled behavior is only available until a BER of $10^{-5}$. After that a similar behavior than the one of the FL can be observed. Due to the nature of the scheduling strategy used for the RL (i.e. best effort), acquiring new resources may be more time demanding, therefore an ARQ block lifetime timeout is more likely with a similar error rate than in the FL.

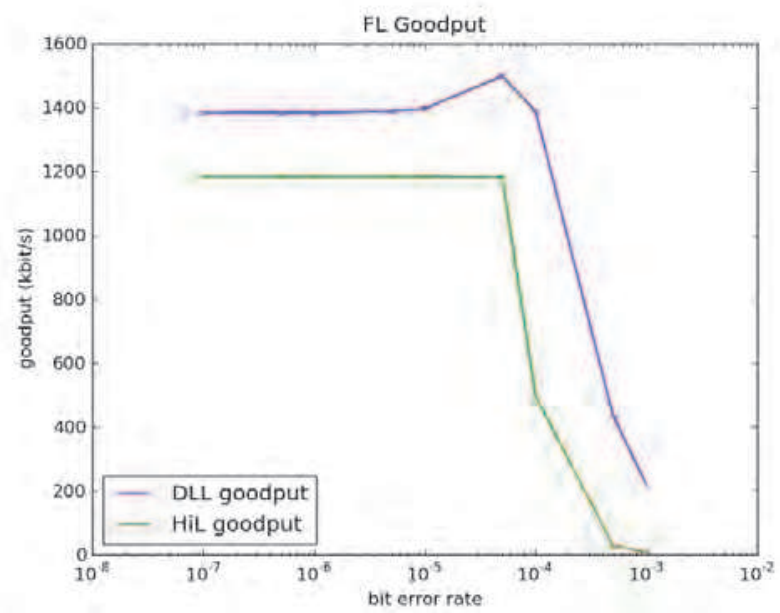

Fig. 18. FL Goodput - scenario BER.

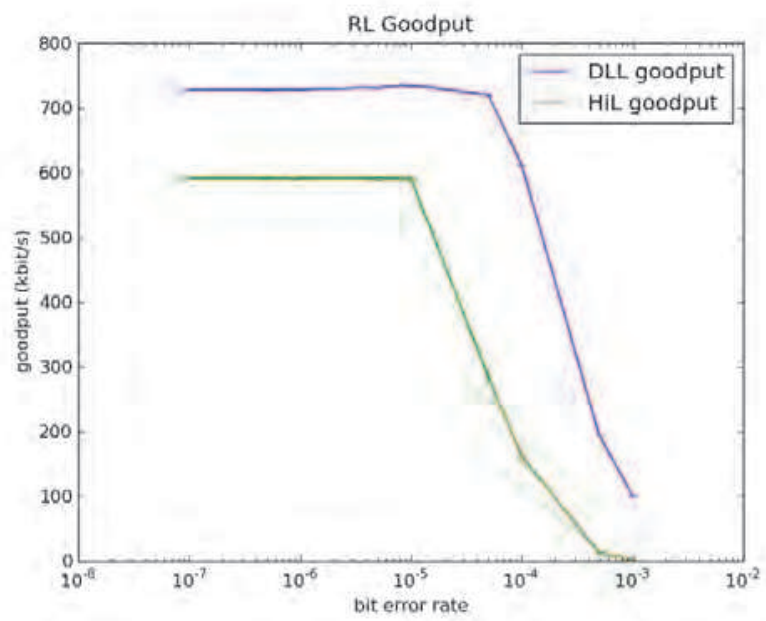

Fig. 19. RL Goodput - scenario BER. 


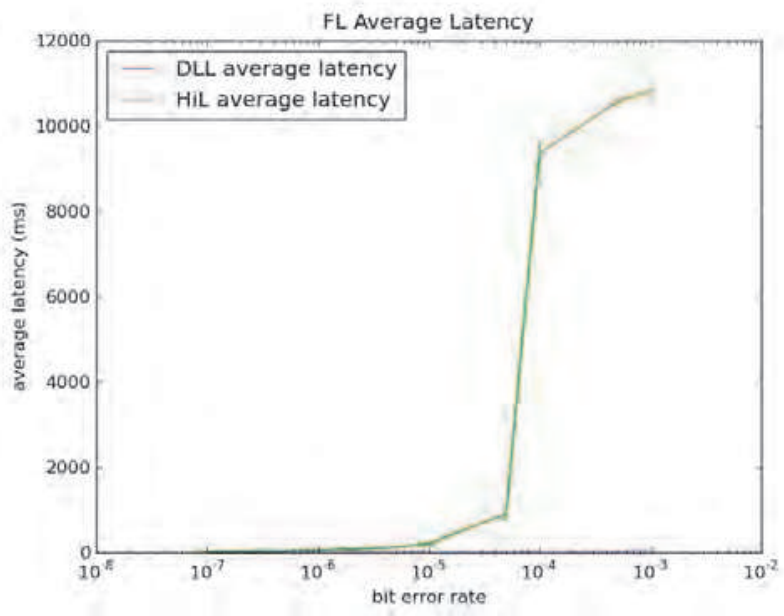

Fig. 20. FL avg. latency - scenario BER.

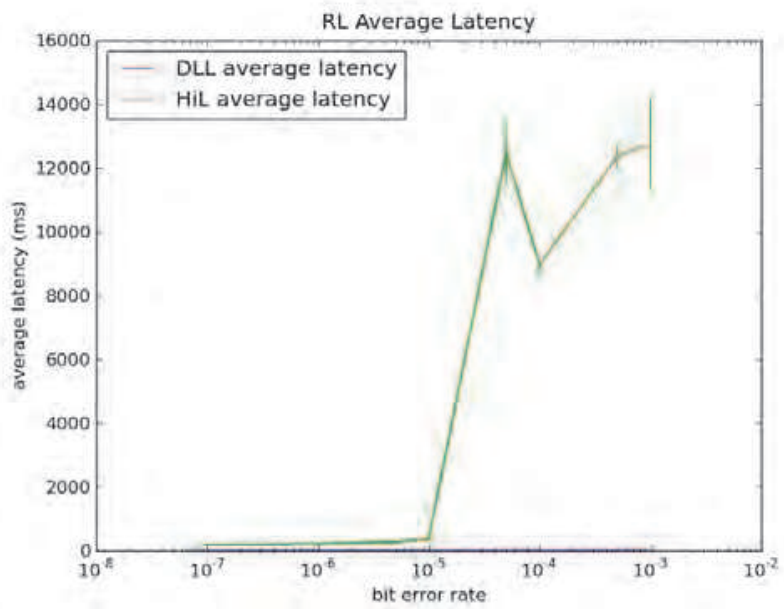

Fig. 21. RL avg. latency - scenario BER.

The LOAD evaluation scenario utilizes exactly the same parameter set than the BER evaluation scenario, except the bit error rate and the data traffic load. The LOAD scenario simulates a rather good channel condition, i.e. BER equal to $10^{-6}$. The purpose of the LOAD scenario is to increase the load in steps starting from $9 \mathrm{kbps}$ per Mobile Station RL data traffic and $18 \mathrm{kbps}$ per Mobile Station FL data traffic. The intervals were set to $2 \mathrm{kbps}$ for the $\mathrm{RL}$ and $4 \mathrm{kbps}$ for the FL. Most load is generated by when $35 \mathrm{kbps}$ for the RL and $70 \mathrm{kbps}$ for the FL are set.

On the FL the higher layer data packets get all through, so do the data link layer packets. The DLL overhead stays proportional for all simulation scenarios. The average FL latency 
increases slightly with more load but is still insignificantly. The RL goodput figure shows an interesting behaviour. Namely the slightly larger DLL goodput with low load. The reason therefore is that with low load resource request are less likely to be piggybacked. Thus, every time a higher layer data packet arrives a separate bandwidth request has to be issued. The overhead is significantly for lower loads as periodic ranging opportunities are accounted for DLL goodput. This overhead stays similar with higher loads, however, relatively the overall overhead decreases. The RL average latency starts to increase when the RL load starts to reach the capacity limit.

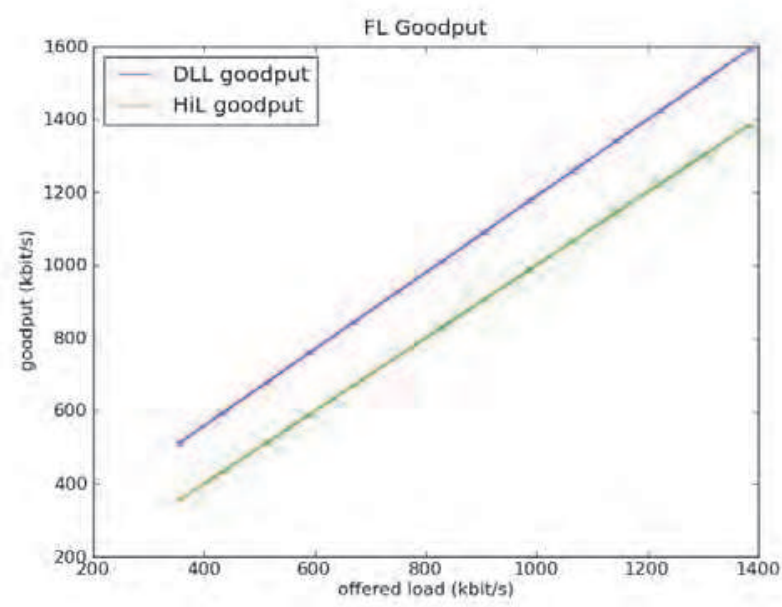

Fig. 22. FL goodput - scenario LOAD.

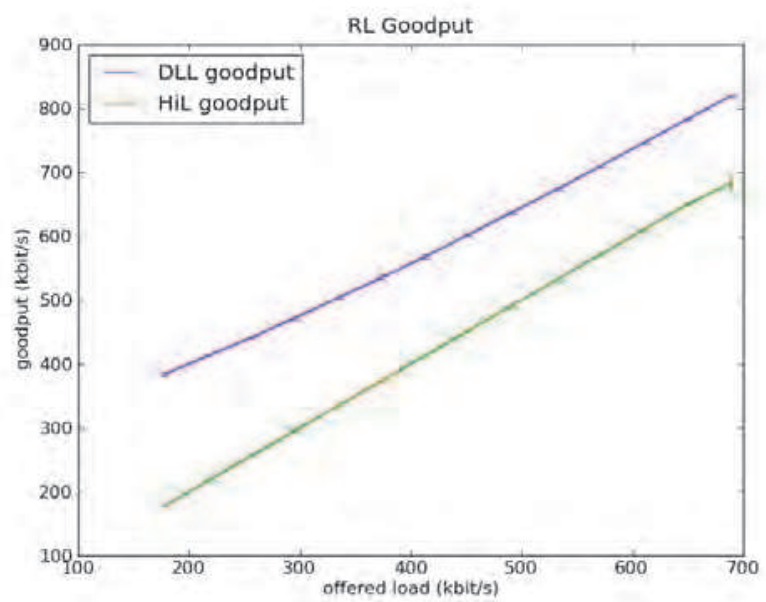

Fig. 23. RL goodput - scenario LOAD. 


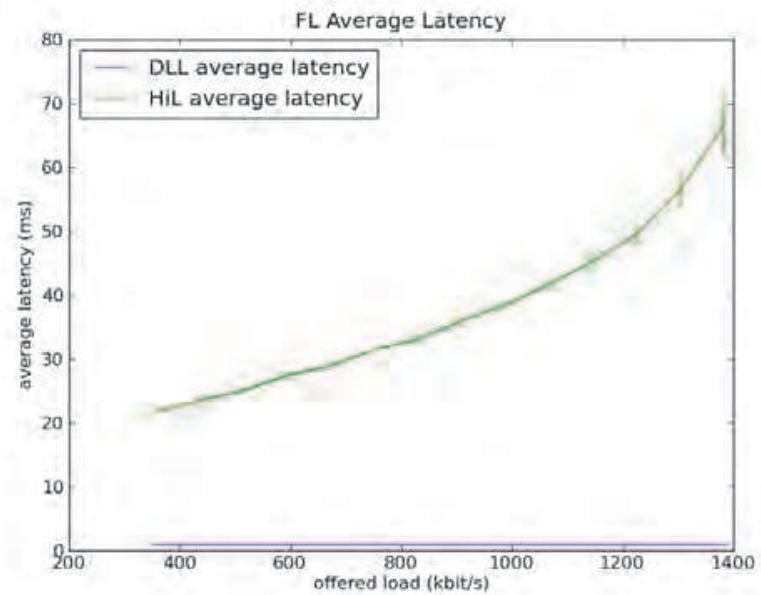

Fig. 24. FL avg. latency - scenario LOAD.

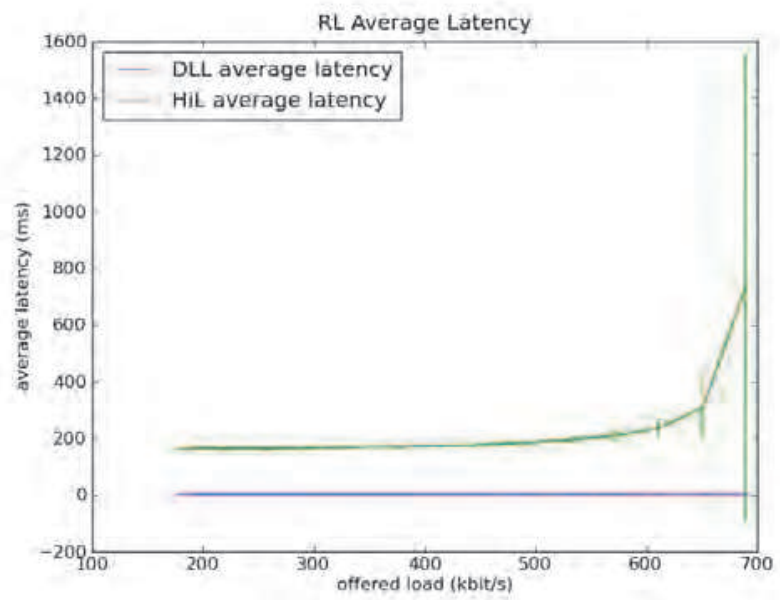

Fig. 25. RL avg. latency - scenario LOAD.

The PIAC (Peak Instantaneous Aircraft Count) evaluation scenario utilizes exactly the same parameter set than the LOAD evaluation scenario, except the amount of Mobile Stations and the data traffic load. The PIAC scenario simulates an average load of $20 \mathrm{kbps}$ per Mobile Station FL data traffic and an average load of $10 \mathrm{kbps}$ per Mobile Station RL data traffic. The amount of Mobile Stations is set to 10 and increased by intervals of 5 up to a maximum value of 80 .

The FL higher layer goodput is reasonable until an amount of 70 concurrent users (and data traffic service flows). After that the throughput decreases and average latency figures increase. The RL higher layer goodput is stable until an amount of 50 concurrent users. However, the data link layer goodput increases with each interval. The reason therefore is 
that bandwidth request ranging opportunities are served with unicast polls. The more concurrent Mobile Stations reside in a single cell, the more overhead is caused through this procedure. The higher layer goodput decreases after more than 50 Mobile Stations are trying to transmit concurrently. The DLL goodput is not reaching its maximum as the RL data subframe gets seriously fragmented through the unicast polls issued by the Base Station. As a result the scheduler gets serious problems utilizing the full spectrum sufficiently. Recall, that ARQ blocks have a fixed size. The average RL latency reflects the ARQ block lifetime.

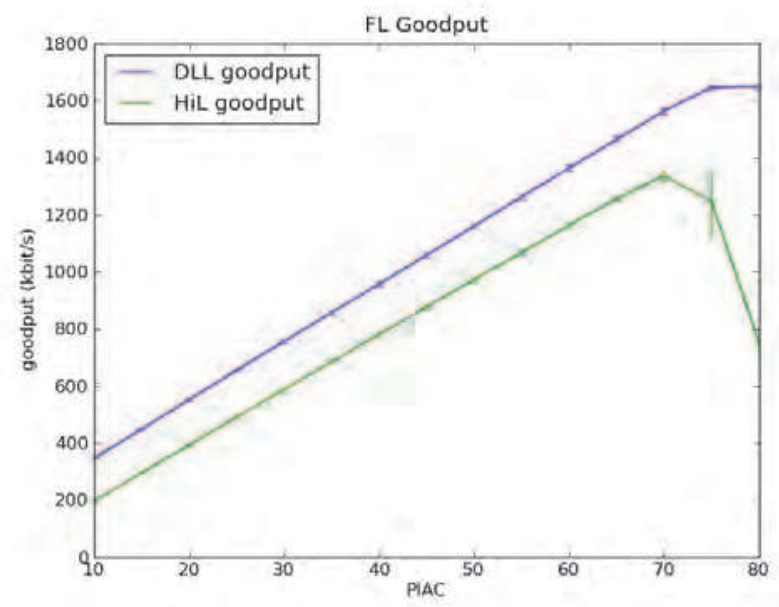

Fig. 26. FL goodput - scenario PIAC.

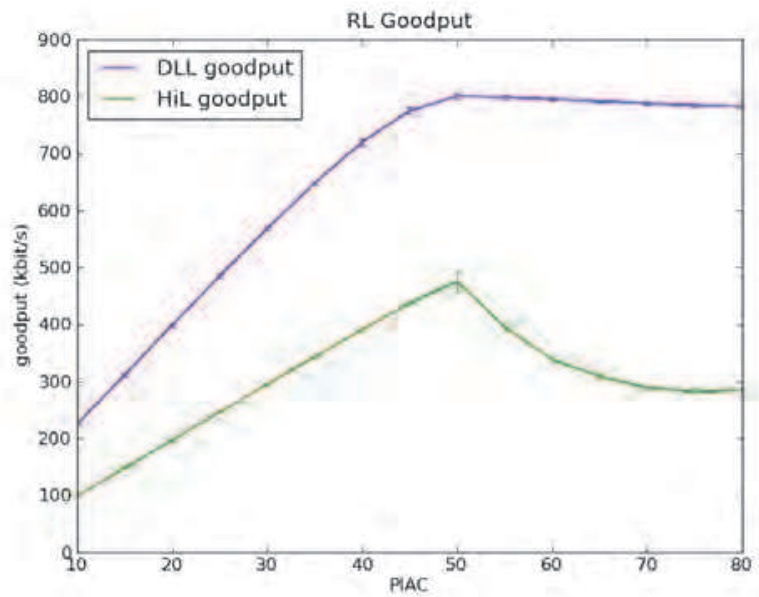

Fig. 27. RL goodput - scenario PIAC. 


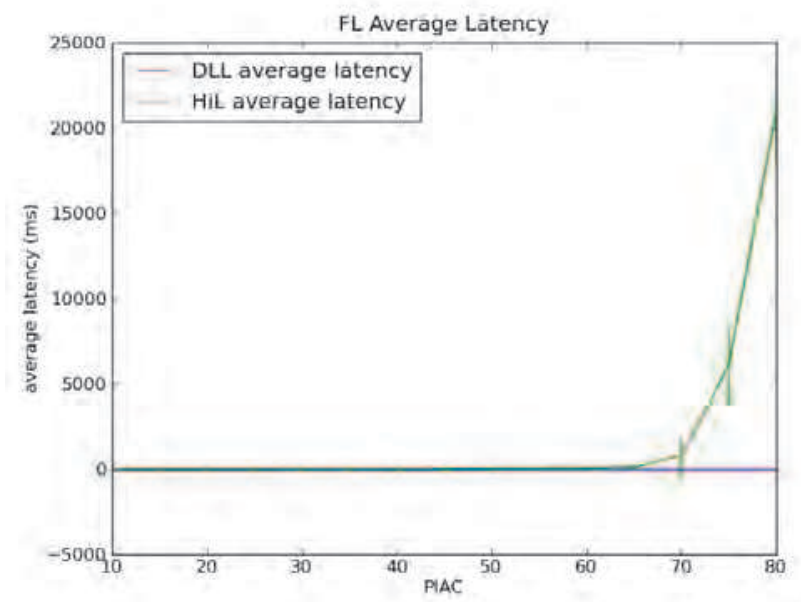

Fig. 28. FL avg. latency - scenario PIAC.

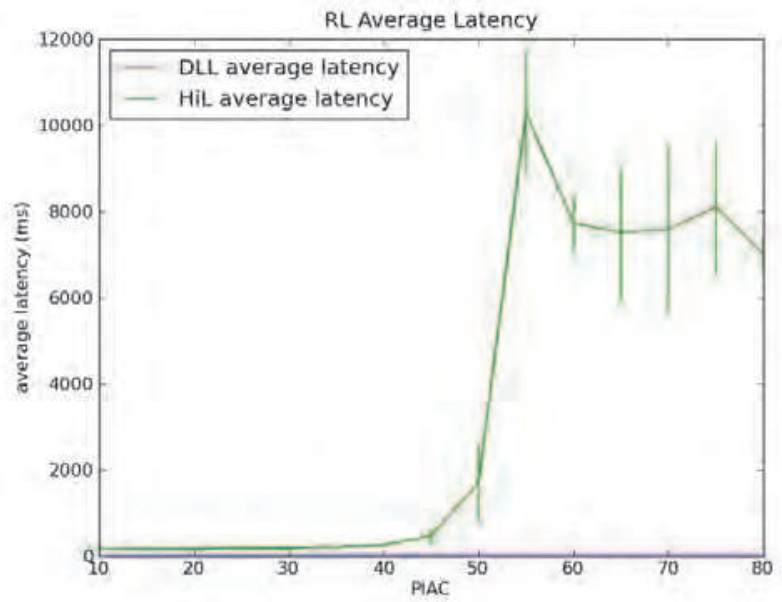

Fig. 29. RL avg. latency - scenario PIAC.

\section{Conclusion}

The demand to integrate the aircraft into the network centric concepts requires capable air ground data-links. AeroMACS shall provide this functionality at the airport surface. Infrastructure and equipage used for aeronautical procedures is evolving very slowly due to several reasons. Cost, interoperability, and safety issues are some among many reasons. Any new system integrated into the aeronautical environment will last for decades until it might eventually be replaced through a new system. Therefore, it is of importance to design new 
systems carefully and with mature concepts in order to remain prepared for changing requirements in the future.

Integrating the AeroMACS sub-network into an IPv6 based aeronautical telecommunication network (ATN) is generally a problem which needs to be resolved from the application's point of view and from the operator point of view. It is also important to keep flexibility in order to be capable to adapt to any future changes of requirements. Especially if products have such long life cycles as in the aeronautical world it is almost impossible to assess the proper requirements.

Multicast applications may be very attractive to the future ATM concept, however, most of these applications are not realized yet (i.e. they exist only in theory). With a wrong sub-net configuration the introduction of application layer concepts based on multicast may be quite difficult and/or expensive.

During the course of the SANDRA project a data traffic load analysis has been conducted which showed that applications with significant load requirements would justify the introduction of a broadband wireless communication system for airport surface communications. Furthermore, MAC performance simulations have shown performance figures to be expected by a future AeroMACS system.

The current status of the AeroMACS profile is a draft. This means that further assessments on the maturity and performance of the technology shall clarify the suitability of AeroMACS for supporting the needs of future ATM concepts. Although currently prototypes are being implemented it is not believed that AeroMACS would be introduced before 2020. A realistic target for the deployment of an AeroMACS system is rather 2025 and beyond.

\section{Acknowledgment}

The research leading to these results has been partially funded by the European Community's Seventh Framework Programme (FP7/2007-2013) under Grant Agreement $n^{\circ}$ 233679. The SANDRA project is a Large Scale Integrating Project for the FP7 Topic AAT.2008.4.4.2 (Integrated approach to network centric aircraft communications for global aircraft operations). The project has 31 partners and started on 1st October 2009.

\section{References}

AOC (2010), M. Wood, S. Lebourg, B. Syren, and P. Huisman, SJU - AOC data-link dimensioning, edition 0.1, 2010.

COCR (2007), Communication Operating Concept and Requirements for the Future Radio System Version 2, May 2007.

Ehammer (2008). M. Ehammer, T. Gräupl, and C.H. Rokitansky, Applying SOA Concepts to the Simulation of Aeronautical Wireless Communication, Spring Simulation MultiConference 2008, April 2008.

Ehammer (2011). M. Ehammer, T. Gräupl, and E. Polo, AeroMACS Data Traffic Model, ICNS 2011, May 2011.

IEEE (2009). IEEE Standard for Local and metropolitan area networks Part 16: Air Interface for Broadband Wireless Access Systems, IEEE Standards Association, May 2011, Available from <http://standards.ieee.org/about/get/802/802.16.html>.

RFC 3315 (2003). Droms et.al, RFC 3315 Dynamic Host Configuration Protocol for IPv6 (DHCPv6), July 2003, status: Proposed Standard, available at 
< http:// tools.ietf.org/html/rfc3315>.

RFC 4291 (2006). Hinden et al., RFC 4291 IP Version 6 Addressing Architecture, February 2006, status: Draft Standard, available at <http://tools.ietf.org/html/rfc4291>

RFC 4861 (2007). T. Narten et al., RFC 4861 Neighbor Discovery for IP version 6 (IPv6), September 2007, status: Draft Standard, available at <http://tools.ietf.org/html/rfc4861>.

RFC 4862 (2007). Thomson et al., RFC 4862 IPv6 Stateless Address Auto-configuration, September 2007, status: Draft Standard, available at <http://tools.ietf.org/html/rfc4862>.

RFC 4903 (2007). D. Thaler, RFC 4903 Multi-Link Subnet Issues, May 2007, status: Informational available at <http:/ / tools.ietf.org/html/rfc4903>.

RFC 5121 (2008). Patil et al., RFC 5121 Transmission of IPv6 via the IPv6 Convergence Sublayer over IEEE 802.16 Networks, February 2008, status: Proposed Standard, available at <http:// tools.ietf.org/html/rfc5121>.

SANDRA. Seamless Aeronautical Networking through integration of Data links, Radios, and Antennas (SANDRA). Large Scale Integrated Project within FP7 - Grant Agreement $\mathrm{n}^{\circ}$ 233679, accessible at <http:/ / www.sandra.aero>.

Sayenko, A.; Tykhomyrov, V.; Martikainen, H. and Alanen, O. (2007). Performance analysis of the ieee 802.16 arq mechanism, Proceedings of the 10th ACM Symposium on Modeling, analysis, and simulation of wireless and mobile systems, ISBN: 978-1-59593851-0, New York, NY, USA.

WiMAX Forum (2009). WiMAX Forum Mobile System Profile Specification: Release 1.5 TDD Specific Part, August 2009.

WiMAX Forum (2010). WiMAX Forum Mobile System Profile DRAFT-T23-R010v09-B; Working Group Approved Revision, May 2010. 


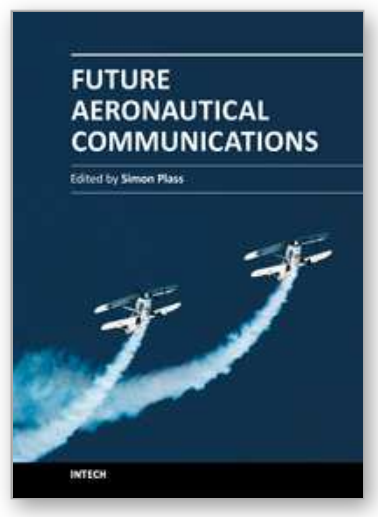

\author{
Future Aeronautical Communications \\ Edited by Dr. Simon Plass
}

ISBN 978-953-307-625-6

Hard cover, 378 pages

Publisher InTech

Published online 26, September, 2011

Published in print edition September, 2011

There are well-founded concerns that current air transportation systems will not be able to cope with their expected growth. Current processes, procedures and technologies in aeronautical communications do not provide the flexibility needed to meet the growing demands. Aeronautical communications is seen as a major bottleneck stressing capacity limits in air transportation. Ongoing research projects are developing the fundamental methods, concepts and technologies for future aeronautical communications that are required to enable higher capacities in air transportation. The aim of this book is to edit the ensemble of newest contributions and research results in the field of future aeronautical communications. The book gives the readers the opportunity to deepen and broaden their knowledge of this field. Todayâ $€^{\mathrm{TM}} \mathrm{S}$ and tomorrowâ€ $€^{\mathrm{TM}} \mathbf{S}$ problems / methods in the field of aeronautical communications are treated: current trends are identified; IPv6 aeronautical network aspect are covered; challenges for the satellite component are illustrated; AeroMACS and LDACS as future data links are investigated and visions for aeronautical communications are formulated.

\title{
How to reference
}

In order to correctly reference this scholarly work, feel free to copy and paste the following:

Max Ehammer, Thomas Gräupl and Elias Pschernig (2011). Utilizing IEEE 802.16 for Aeronautical Communications, Future Aeronautical Communications, Dr. Simon Plass (Ed.), ISBN: 978-953-307-625-6, InTech, Available from: http://www.intechopen.com/books/future-aeronautical-communications/utilizing-ieee802-16-for-aeronautical-communications

\section{INTECH}

open science | open minds

\section{InTech Europe}

University Campus STeP Ri

Slavka Krautzeka 83/A

51000 Rijeka, Croatia

Phone: +385 (51) 770447

Fax: +385 (51) 686166

www.intechopen.com

\section{InTech China}

Unit 405, Office Block, Hotel Equatorial Shanghai

No.65, Yan An Road (West), Shanghai, 200040, China

中国上海市延安西路65号上海国际贵都大饭店办公楼 405 单元

Phone: +86-21-62489820

Fax: $+86-21-62489821$ 
(C) 2011 The Author(s). Licensee IntechOpen. This chapter is distributed under the terms of the Creative Commons Attribution-NonCommercialShareAlike-3.0 License, which permits use, distribution and reproduction for non-commercial purposes, provided the original is properly cited and derivative works building on this content are distributed under the same license. 